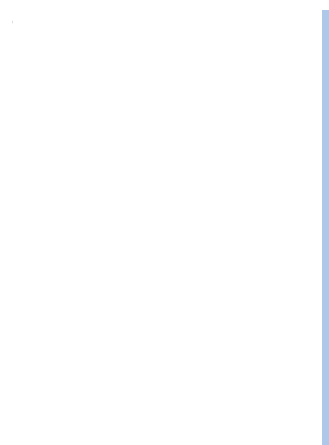

Journal of Geology,

Geography and

Geoecology

ISSN 2617-2909 (print)

ISSN 2617-2119 (online)

Journ. Geol. Geograph. Geology,

30(4), 692-705.

Journal home page: geology-dnu.dp.ua

doi: $10.15421 / 112164$

Mammadov M.N., Babayeva G.J.

Journ. Geol. Geograph. Geoecology, 30(4), 692-705

\title{
Petrogenetic peculiarities of Fe-Ti oxide minerals in the processes of crystallization and evolution of late Cretaceous volcanic complexes of the Lesser Caucasus
}

\author{
Musa Nasib ogly Mammadov, Gultekin Javad gizi Babayeva \\ Institute of Geology and Geophysics of the National Academy of Sciences of Azerbaijan, Baku, Azerbaijan Republic, \\ gultekin56@rambler.ru
}

\section{Received: 05.03.2021 \\ Received in revised form: 19.07.2021 \\ Accepted: 10.08 .2021}

\begin{abstract}
Drawing from the determined differences between iron-titanium oxide minerals, we analyzed the conditions of crystallization and evolution of late-Cretaceous magmatic complexes of the Lesser Caucasus. It was found that the rocks of basalt-andesibasalt complex, which correspond to the early substage (upper Coniacian-lower Santonian) of
\end{abstract} late-Cretaceous volcanism in the Qazakh, Agjakand, Agdara depressions, have crystallized in the conditions of relatively highlythermobaric crystallization of titanomagnetite, poorly differentiated and evolutionized according to the Fenner trend. In the second substage of volcanism, due to decrease in permeability of the Earth's crust, the elevation of the remaining magma to the upper horizons was hindered. Therefore, within the Qazakh depression, shallow intermediate sites of crystallization developed where moderately titaniferous magnetite crystallized with the participation of oxidized fluids earlier than hornblende, pyroxene and plagioclase. Thus, the remaining magma evolutionized its composition through Bowen's reaction series. In the Agjakand and Agdara depressions, change of previous expansion to compaction was the cause of hindering of partly fractioned portion of the magma. The latter thermally interacted with the above-embedded maghemite, hematite and in a number of cases magnetite. In the Khojavand depression, rocks of trachibasalttrachiodolerite complex, which characterize the late substage of the Santonian volcanism, contain moderately titanium magnetites and maghemites. In the second substage of volcano-plutonism, rocks of tephrite-teshenite complex developed. There, accompanied by oxidized fluids, highly-clayey titanomagnetite crystallized before chrome-diopside and salite. However, the ulvospinel titanomagnetite in teshenites, having associated with barkevikite and kaersutite, crystallized at a relatively higher temperature. Within the Senonian volcanites of the Azykh depression, along with the moderately-titanium magnetite, chromic titanomagnetite and rarely chromite was determined. Similar mineralogical diversities are also characteristic for the Gochas depression.

Keywords: Lesser Caucasus, Fe-Ti oxide minerals, magnetite-ilmenite thermometer, Curie point, crystallization differentiation

\section{Петрогенетичні особливості Fе-Ті оксидних мінералів у процесі кристалізації та еволюції пізньокрейдових вулканічних комплексів Малого Кавказу}

\author{
М.Н. Мамедов, Г.Д. Бабаєва
}

Інститут геології та геофізики НАН Азербайджану, Азербайджанська республіка, м. Баку, gultekin56@rambler.ru

Анотація. У статті, на підставі встановлених відмінностей залізо-титан оксидних мінералів, аналізуються умови кристалізації та еволюційного перетворення пізньокрейдових магматичних комплексів Малого Кавказу. Виявлено, що породи базальтандезібазальтового комплексу, що відповідають ранньому підетапу (верхній коньяк-нижній сантон) пізньокрейдового вулканізму Газахського, Агджакендського і Агдеринського прогинів, кристалізувалися при порівняно високотермобаричної кристалізації титаномагнетиту, слабодиференційований і еволюціонований Феннерівським шляхом. У другому підетапі вулканізму, у зв'язку з зменшенням проникності земної кори, відбувалося утруднення підйому залишкового розплаву у верхні горизонти. Тому в межах Газахського прогину формувалися малоглибинні проміжні вогнища, де при супроводі окислених флюїдів відбувалася кристалізація помірно-титаністого магнетиту раніше рогової обманки, піроксену і плагіоклазу. У цьому залишковий розплав еволюціонував свій склад Боуеновским шляхом. У Агджакендском і Агдеринском прогинах зміна попереднього розтягнення на стиск стала причиною затримки частково відфракціонованої порції розплаву. Останній термічно взаємодіяв з лежачими аркозовими пісковиками, у результаті формувалися корові дацити і риодацити, у складі яких беруть участь маггеміт, гематит та іноді магнетит. У Ходжавендскому прогині породи трахибазальт-трахидолеритового комплексу, що характеризують ранній підетап сантонського вулканізму, містять помірно-титанисті магнетити і маггеміти. У другому підетапі вулкано-плутонізму відбувалося формування порід тефрит-тешенитового комплексу. Тут, при супроводі окислених флюїдів, високоглиноземистий титаномагнетит кристалізувався раніше хромдіопсіду і саліту. Однак у складі тешенітів ульвошпінелевий титаномагнетит, тісно асоціюючи з баркевікітом і керсутитом, кристалізувався при порівняно більш високій 
температурі. У складі сенонських вулканітів Азихського прогину поряд з помірно-титаністим магнетитом встановлено хромистий титаномагнетит і рідко хроміт. Подібні мінералогічні розмаїття характерні й у Гочасського прогині.

Ключові слова: Малий Кавказ, Fе-Ті оксидні мінерали, магнетит-ільменітовий термометр, точка Кюрі, кристалізаиійна диференціачія.

\section{Introduction.}

Iron-titanium oxide minerals that crystallized at different-depth intermediate sites and intrusive chambers bear a certain petrogenetic data, particularly location of deposits in series of crystallization, structuraloptical types, chemical and mineral compositions, and petromagnetic peculiarities of iron-titanium oxide minerals.

The abovementioned peculiarities of these minerals can be used for clarifying physical-chemical $\left(\mathrm{P}, \mathrm{T}, \mathrm{fo}_{2}\right)$ and geodynamic conditions (compression, expansion) of crystallization of magmatic rocks.

To determine the temperature and partial pressure of oxygen of crystallization of iron-titanium oxide minerals as a geological thermometer, the following methods are used: Buddington-Lindsley (Buddington, Lindsley, 1964), Carmichael (Carmichael,1965), Anderson (Anderson, 1968), Lindsley et al. (Lindsley, Spencer, 1982), Stormer (Stormer, 1983), Genshaft et al. (Genshaft et al., 1999, 2003), and to determine depths of crystallization - methods of Kawai (Kawai, 1956), Osborn (Osborn, 1983), Pechetskij (Pecherskij, 1975, 1985), Zubov et al. (Zubov i dr., 2015).

Based on the interpretation of thermomagnetic and chemical analyses of titanomagnetites and ilmenites, Creek and Ibbetson (Creek, Ibbetson, 1970), and D. M. Pecherskij (Pecherskij, 1975) propose using experimental and calculated values of Curie points.

Along with them, the location of titanomagnetite crystallization, according to Osborn (Osborn, 1983), is an indicator of oxidative and reduction conditions of the evolutionary conversion of magmatic melts.

In this article, we attempted to determine the physical-chemical and geological conditions of crystallization of rocks of late Cretaceous magmatic complexes of the Lesser Caucasus according to the noted peculiarities of iron-titanium oxidative minerals.

\section{Methods of studies.}

With the purpose of determining significance of the presented analyses of iron-titanium oxide minerals, we used microprobe, chemical, X-ray diffractometer and thermomagnetic methods.
Microprobe analyses of impregnations with FeTi oxide minerals, chrome-spinels and olivines were carried out in a electron-microprobe analyzer (JEOL, JSM-6610 LV, Oxford Instruments, X-MAX) with internal standard. All the microprobe, X-ray diffractionmetric and chemical analyses of iron-titanium oxide minerals were performed at the Analytical Center of the Institute of Geology and Geophysics of the National Academy of Sciences of Azerbaijan.

Monomineral fractions of rocks (weighing 20 $\mathrm{kg}$ ) were divided at the Institute of Geology and Geophysics of the Siberian Branch of the Russian Academy of Sciences (SB RAS), under the leadership of T.S. Yusupova. For the chemical analysis, we used fractions sized $0.25 \mathrm{~mm}$ and purity was tested with binocular magnifying glass.

During the calculation of Curie points, we used chemical and microprobe analyses of $\mathrm{TiO}_{2}$ (Nagata, 1965; Pecherskij, 1975).

Crystal chemical formulae of minerals and evaluations of the conditions of their development $(\mathrm{T}$, $\mathrm{fo}_{2}$ ) were calculated in ILMAT software (Lepage, 2003).

Geological and petrogenetic peculiarities of the Late Cretaceous magmatic complexes of the Lesser Caucasus. For the purpose of clarifying the depths of localization, and therefore the conditions of crystallization of the rocks, the most expedient objects were considered to be the Late Cretaceous magmatic complexes of the Lok-Garabagh (Gazakh, Agjakand, Agdara depressions), Goycha-Akeri (Khojavand, Azykh depressions) and Miskhan-Kafan (Gochas depression) structural-formation zones of the Lesser Caucasus (Shikhalibejli, 1994) (Fig.1).

The Lok-Garabagh structural-formation zone. This zone, starting from the Lok crystalline structure, in parallel to the southern side of the Kura depression, runs eastward from the west in the general-Caucasian direction to the Murovdagh thrust fault. From the southwest contour of the indicated thrust fault, it changes its strike toward the southeast and is seen to the middle reach of the Khachinchay River.

The Late Cretaceous volcanic complexes of the analyzed zone within Azerbaijan are developed in the Gazakh, Agjakand, Agdara depressions. 


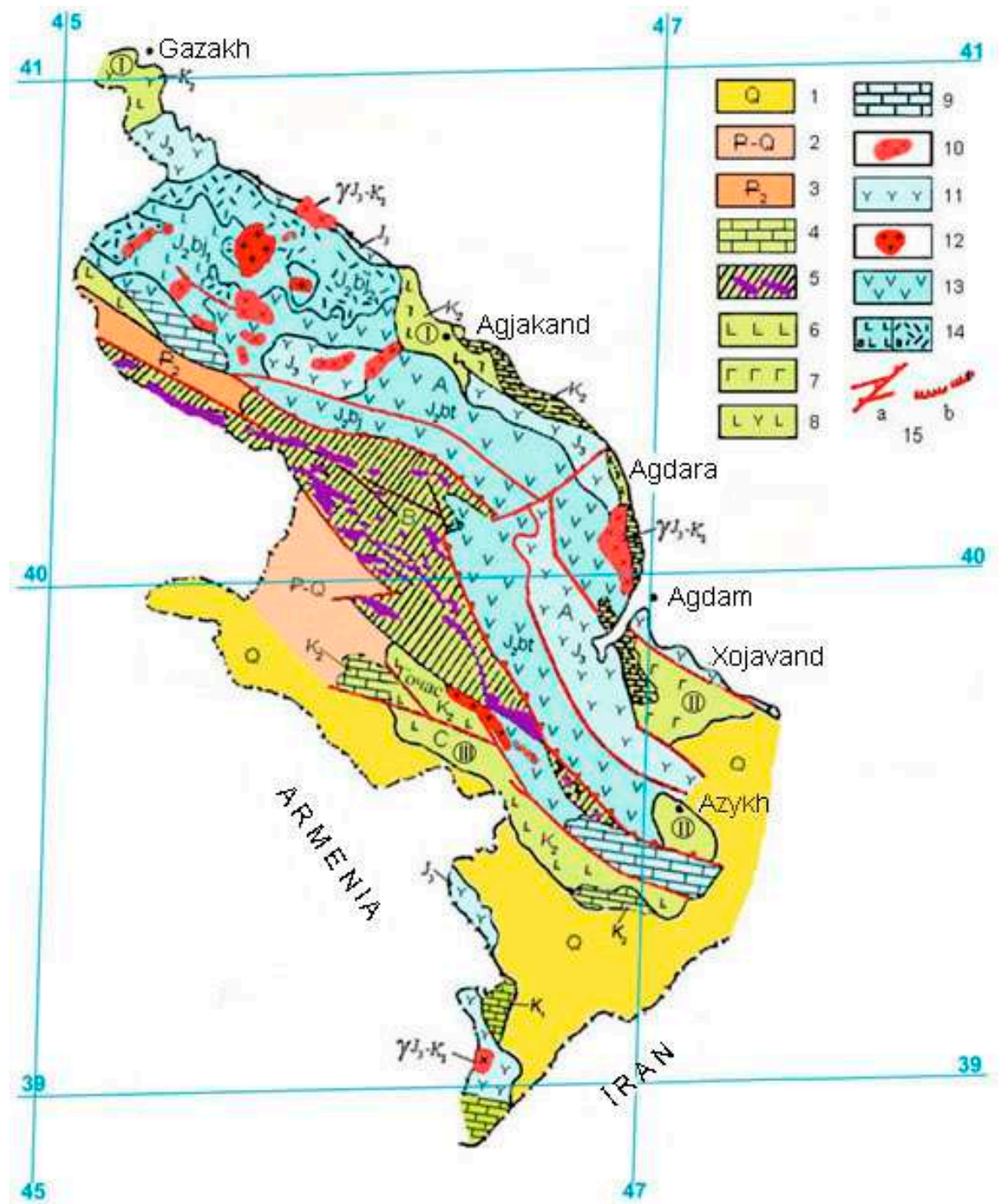

Fig. 1. Schematic geological map of the Lesser Caucasus (Shikhalibejli, 1994).

I-Lok-Garabagh structural-formation zone (Gazakh, Agjakand, Agdara depressions); II - Goycha-Akeri structural-formation zone (Azykh and Khojavand depressions), III-Miskhan-Kafan structural-formation zone (Gochas depression)

1 -Contemporary sediments; 2 - Paleogene-Neogene volcanogenic sedimentary deposits; 3 -Paleogene volcanogenic sedimentary deposits; 4 - Upper Cretaceous limestones; 5 - ophiolite complexes; 6 - Late Senonian basalt-andesibasalt and trachybasalt-trachyandesibasalt complexes (Gochas depression); 7 - Santonian basalt-andesibasalt and trachybasalt-trachyandesibasalt complexes (Azykh depression), trachybasalt-trachydolerite and tephrite-teschenite (Khojavand depression) complexes; 8 - Late Coniacian-Early Santonian basaltandesibasalt and Late Santonian-Early Campanian rhyolite-rhyodacite complexes (Gazakh depression), Coniacian- Santonian basaltandesibasalt and rhyolite-rhyodacite complexes (Agjakand and Agdara depressions); 9 - Upper Jurassic limestones; 10 - Late Jura-Early Cretaceous gabbro-diorite-granite complex; 11 - late Jurassic-early Cretaceous dacite complex; 12 - Bathonian plagiogranite complex; 13 - Bathonian basalt-andesite-dacite-rhyoilte complex; 14 -a) Early Bajocian basalt complex, б) late Bajocian rhyolite complex; 15 deep faults (a), flexures (b).

Based on the petrographic composition and time of emergence of volcanism, in correspondence with the stage of volcanic activity, the Late Cretaceous volcanites of the Gazakh depression are divided into two following complexes: 1) basalt-andesibasalt; 2) rhyolite-rhyodacite (Mamedov, 1999) (Table 1).

The first complex that characterizes the substage of volcanism (the Upper Coniacian-Lower Santonian), is mainly composed of volcanic breccias, currents, thin sill and dykes of dolerites, basalts, andesibasalts and also a subordinate number of andesites.
The second complex corresponds to the Late Cretaceous substage (the Upper Santonian-Early Campanian) of volcanism and is represented by rhyolite, rhyodacite extrusive rocks and zeolitic tuff.

In the central part of the Gazakh depression, the composition of the first complex is noticeably dominated by lava flows, lava breccias of olivine, olivine-clinopyroxene and clinopyroxene-plagioclase basalts. In the southwest outskirts of the depression, there are thin flows of olivine-chrome-spinel picrites and olivine-clinopyroxene picrobasalts. Within the 


\begin{tabular}{|c|c|c|c|c|c|c|c|c|c|c|c|c|c|c|}
\hline \multirow{2}{*}{ 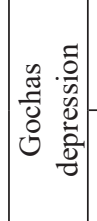 } & әң!૬әривКцәв.п & $\begin{array}{l}\text { ते } \\
\text { ڤn }\end{array}$ & $\stackrel{\text { In }}{\rightarrow}$ & $\begin{array}{l}\bar{n} \\
\underline{0}\end{array}$ & $\underset{m}{\bar{m}}$ & $\stackrel{\infty}{-\infty}$ & $\begin{array}{l}\infty \\
\stackrel{0}{0}\end{array}$ & $\stackrel{\bullet}{\vec{i}}$ & $\stackrel{े}{\vec{n}}$ & $\begin{array}{l}\text { m. } \\
\text { in }\end{array}$ & $\stackrel{i}{n}$ & $\overrightarrow{\tilde{o}}$ & $\stackrel{\circ}{\circ}$ & $\begin{array}{l}\text { గొ } \\
\stackrel{2}{\alpha}\end{array}$ \\
\hline & H[eseq & 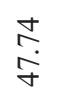 & $\stackrel{?}{\stackrel{2}{2}}$ & $\stackrel{\infty}{\stackrel{\infty}{ٍ}}$ & $\vec{\sigma}$ & $\underset{i n}{+}$ & $\begin{array}{l}0 \\
\stackrel{0}{0}\end{array}$ & $\begin{array}{l}\tilde{\sigma} \\
\text { in }\end{array}$ & $\stackrel{m}{0}$ & $\begin{array}{l}\hat{\sigma} \\
\dot{n}\end{array}$ & $\hat{a}$ & $\tilde{n}$ & $\stackrel{\text { సे }}{i}$ & 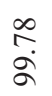 \\
\hline \multirow{2}{*}{ 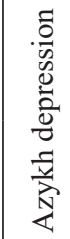 } & 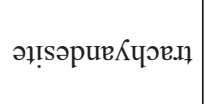 & $\begin{array}{l}\text { స్ } \\
\stackrel{n}{n}\end{array}$ & $\stackrel{0}{=}$ & 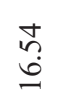 & ర్ర & $\stackrel{શ}{I}$ & $\vec{\jmath}$ & $\stackrel{\circ}{n}$ & $\stackrel{\stackrel{f}{+}}{\stackrel{f}{*}}$ & ơ & $\stackrel{\widehat{N}}{\widehat{N}}$ & $\stackrel{5}{\circ}$ & $\stackrel{n}{i}$ & $\begin{array}{l}\hat{n} \\
\alpha \\
\alpha\end{array}$ \\
\hline & H[sseq & $\begin{array}{l}8 \\
\stackrel{8}{\circ} \\
\text { in }\end{array}$ & $\stackrel{?}{\stackrel{i}{-}}$ & 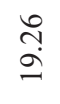 & $\begin{array}{l}: \\
\dot{r}\end{array}$ & $\underset{\text { ָิ }}{+}$ & $\stackrel{े}{\circ}$ & $\stackrel{\overbrace{}}{+}$ & ֶิ & $\begin{array}{l}\infty \\
i \\
i\end{array}$ & $\begin{array}{l}0 \\
\infty \\
0 \\
0\end{array}$ & $\tilde{\tilde{o}}$ & $\stackrel{\infty}{i}$ & $\begin{array}{l}\overrightarrow{6} \\
\stackrel{\alpha}{ }\end{array}$ \\
\hline \multirow{4}{*}{ 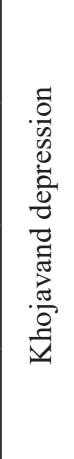 } & 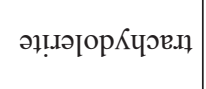 & $\begin{array}{l}\hat{6} \\
\dot{f}\end{array}$ & $\stackrel{\circ}{\circ}$ & $\begin{array}{l} \pm \\
\dot{ \pm}\end{array}$ & $\underset{ָ}{\sim}$ & $\begin{array}{l}\vec{b} \\
\dot{n}\end{array}$ & ণิ & $\stackrel{n}{r}$ & 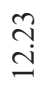 & $\stackrel{\text { กै }}{+}$ & $\stackrel{\text { gे }}{-}$ & $\stackrel{0}{0}$ & $\begin{array}{l}\vec{b} \\
i\end{array}$ & $\begin{array}{l}\hat{2} \\
\stackrel{\alpha}{\alpha}\end{array}$ \\
\hline & HеSeq Кчગ્.I & $\frac{n}{j}$ & 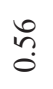 & $\begin{array}{l}\stackrel{R}{~} \\
i n\end{array}$ & $\stackrel{n}{\stackrel{0}{0}}$ & $\underset{i}{\stackrel{J}{*}}$ & $\stackrel{?}{\circ}$ & $\frac{\infty}{6}$ & $\stackrel{\sim}{\sim}$ & $\stackrel{\overbrace{}}{\sim}$ & $\stackrel{\triangleright}{\circledR}$ & $\stackrel{\ddot{g}}{=}$ & $\stackrel{\text { }}{\text { i }}$ & $\stackrel{m}{\alpha}$ \\
\hline & 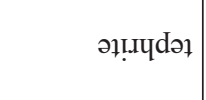 & $\begin{array}{l}\vec{J} \\
\dot{J}\end{array}$ & $\stackrel{\tilde{\rho}}{-}$ & $\vec{m}$ & $\underset{\hat{\sigma}}{\hat{A}}$ & $\stackrel{m}{m}$ & $\stackrel{2}{\circ}$ & $\stackrel{m}{\infty}$ & $\begin{array}{l}\stackrel{\&}{\mathrm{I}} \\
\stackrel{\mathrm{I}}{ }\end{array}$ & $\stackrel{\infty}{\stackrel{\leftrightarrow}{+}}$ & $\begin{array}{l}\vec{\infty} \\
\stackrel{0}{0}\end{array}$ & $\stackrel{\mathbb{t}}{\stackrel{0}{0}}$ & $\begin{array}{l}\text { ț } \\
i\end{array}$ & $\begin{array}{l}0 \\
\text { } \\
\text { à }\end{array}$ \\
\hline & 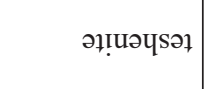 & $\begin{array}{l}\tilde{y} \\
\text { j }\end{array}$ & 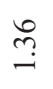 & 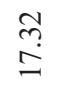 & $\underset{i}{\stackrel{8}{i}}$ & ले & $\stackrel{m}{0}$ & ف. & 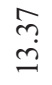 & $\stackrel{=}{m}$ & f̊. & $\stackrel{+}{m}$ & $\stackrel{m}{\dot{n}}$ & $\begin{array}{l}\text { ర్ } \\
\infty \\
\infty\end{array}$ \\
\hline \multirow{5}{*}{ 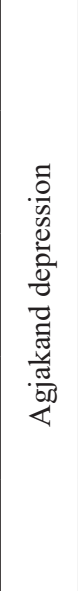 } & 라!|디 & $\begin{array}{l}\stackrel{0}{0} \\
\infty\end{array}$ & $\stackrel{\rightleftarrows}{=}$ & $\begin{array}{l}\infty \\
i \\
i\end{array}$ & $\begin{array}{l}\stackrel{\sim}{N} \\
\text { in }\end{array}$ & $\begin{array}{l}n \\
0 \\
0\end{array}$ & 苛 & $\stackrel{?}{\circ}$ & $\stackrel{8}{\stackrel{\circ}{+}}$ & $\hat{\tilde{m}}$ & $\stackrel{\text { ঙิ }}{-}$ & $\stackrel{\infty}{0}$ & $\stackrel{\bar{i}}{i}$ & $\begin{array}{l}\text { के } \\
\stackrel{\alpha}{ }\end{array}$ \\
\hline & \multirow{2}{*}{ Syfeseq } & 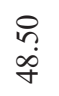 & $\stackrel{\infty}{\circ}$ & 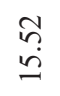 & $\underset{\sim}{\tilde{r}}$ & $\begin{array}{l}\stackrel{+}{~} \\
\text { in }\end{array}$ & $\stackrel{0}{\circ}$ & f & 苂 & $\underset{f}{\stackrel{f}{f}}$ & $\stackrel{n}{\text { Iq }}$ & $\stackrel{\ddot{g}}{=}$ & $\stackrel{?}{=}$ & $\begin{array}{l}\text { f) } \\
\stackrel{2}{\alpha}\end{array}$ \\
\hline & & $\begin{array}{l}0 \\
\dot{y}\end{array}$ & $\begin{array}{l}0 \\
\infty \\
0\end{array}$ & $\begin{array}{l}\stackrel{f}{+} \\
\dot{ \pm}\end{array}$ & $\begin{array}{l}\stackrel{0}{0} \\
\text { in }\end{array}$ & $\frac{n}{a}$ & $\stackrel{0}{\stackrel{0}{0}}$ & $\stackrel{\leftrightarrow}{\text { co }}$ & 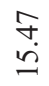 & $\vec{i}$ & $\stackrel{\widetilde{m}}{\sim}$ & $\stackrel{\check{I}}{\leftrightarrows}$ & $\stackrel{n}{i}$ & $\begin{array}{l}\hat{\Upsilon} \\
\text { ă }\end{array}$ \\
\hline & \multirow{2}{*}{ səł!.ણ૦ор } & 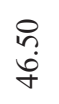 & $\stackrel{\tilde{\rho}}{-}$ & $\begin{array}{l}\stackrel{\$}{2} \\
\stackrel{-}{2}\end{array}$ & $\begin{array}{l}\hat{\sigma} \\
\dot{+}\end{array}$ & ळे & $\stackrel{ \pm}{0}$ & $\bar{\sigma}$ & $\stackrel{q}{\stackrel{f}{=}}$ & 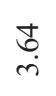 & $\underset{-}{\sigma}$ & $\stackrel{\vec{I}}{=}$ & $\stackrel{+}{\stackrel{2}{2}}$ & $\begin{array}{l} \pm \\
\stackrel{\circ}{\alpha}\end{array}$ \\
\hline & & $\begin{array}{l}0 \\
\text { లె. } \\
\dot{f}\end{array}$ & $\begin{array}{l}\infty \\
\stackrel{0}{0}\end{array}$ & $\begin{array}{l}n \\
\ddot{0} \\
\varrho\end{array}$ & 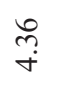 & $\stackrel{\infty}{\stackrel{\infty}{r}}$ & ઼ָ & f̊ & $\begin{array}{l}\infty \\
\stackrel{\infty}{త} \\
\end{array}$ & $\stackrel{m}{m}$ & $\stackrel{\vec{\sigma}}{-}$ & $\stackrel{\check{I}}{\sharp}$ & $\stackrel{\overbrace{}}{\overparen{T}}$ & $\begin{array}{l}\text { +ั. } \\
\text { aे }\end{array}$ \\
\hline \multirow{5}{*}{ 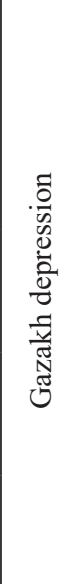 } & ગૈ!| & $\stackrel{\text { f }}{\stackrel{2}{8}}$ & $\overrightarrow{\widetilde{o}}$ & $\begin{array}{l}\stackrel{0}{1} \\
\stackrel{n}{n}\end{array}$ & $\stackrel{d}{\underset{-}{*}}$ & $\stackrel{f}{\rightarrow}$ & $\stackrel{\infty}{\circ}$ & $\begin{array}{l}\infty \\
\stackrel{n}{0}\end{array}$ & $\underset{m}{=}$ & $\stackrel{\dddot{q}}{\dot{r}}$ & $\underset{\sim}{\exists}$ & $\stackrel{\check{I}}{=}$ & $\stackrel{n}{0}$ & $\begin{array}{l}\infty \\
\infty \\
\alpha\end{array}$ \\
\hline & \multirow{2}{*}{ Słjeseq } & $\begin{array}{l}\stackrel{0}{0} \\
\stackrel{+}{q}\end{array}$ & $\underset{-}{8}$ & $\begin{array}{l}\infty \\
\infty \\
\stackrel{\sim}{~}\end{array}$ & $\begin{array}{l}\vec{n} \\
i n\end{array}$ & 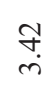 & $\stackrel{\infty}{\circ} \stackrel{0}{\circ}$ & $\stackrel{7}{m}$ & $\stackrel{\stackrel{n}{:}}{=}$ & 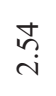 & లి & $\stackrel{\vec{I}}{=}$ & ㄱ. & aे \\
\hline & & $\frac{\mathbb{N}}{\stackrel{q}{q}}$ & $\stackrel{5}{0}$ & $\underset{\stackrel{\Delta}{a}}{\stackrel{\Delta}{a}}$ & $\hat{r}$ & $\hat{n}$ & $\stackrel{\circ}{\circ}$ & $\stackrel{+}{\stackrel{+}{+}}$ & $\stackrel{\text { సे }}{=}$ & तָ & $\stackrel{ \pm}{0}$ & $\stackrel{\ddot{g}}{=}$ & $\begin{array}{l}\text { + } \\
0 \\
0\end{array}$ & $\begin{array}{l}\vec{\sim} \\
\stackrel{8}{0}\end{array}$ \\
\hline & \multirow{2}{*}{ sәң!..ણ૦ор } & $\begin{array}{l}n \\
0 \\
b \\
+\end{array}$ & $\stackrel{\infty}{\circ}$ & 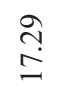 & $\stackrel{\text { nे }}{r}$ & $\overrightarrow{\widehat{\sigma}}$ & $\stackrel{\circ}{\circ}$ & 节 & $\underset{f}{\exists}$ & $\stackrel{0}{\vec{i}}$ & $\stackrel{\infty}{\circ}$ & $\stackrel{\vec{j}}{\dot{I}}$ & $\ddot{\Xi}$ & $\begin{array}{l}: \\
: \\
\circ\end{array}$ \\
\hline & & $\begin{array}{l}8 \\
\dot{f}\end{array}$ & $\stackrel{\tilde{o}}{-}$ & $\stackrel{n}{\stackrel{n}{I}}$ & $\begin{array}{c}\tilde{n} \\
\hat{n}\end{array}$ & 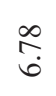 & $\stackrel{7}{\circ}$ & $\underset{\sim}{\stackrel{J}{r}}$ & $\stackrel{\text { fo }}{=}$ & $\widehat{\widehat{\Lambda}}$ & $\stackrel{\infty}{n}$ & $\stackrel{\ddot{g}}{=}$ & 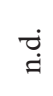 & $\begin{array}{l}\hat{\sim} \\
\text { à }\end{array}$ \\
\hline 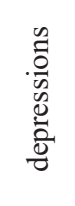 & & $\oslash^{N}$ & $\stackrel{\curvearrowright}{\circledast}^{N}$ & $\stackrel{0^{n}}{\underset{\leftarrow}{n}}$ & $\begin{array}{l}0^{\infty} \\
\omega^{\infty}\end{array}$ & $\begin{array}{l}8 \\
\\
1\end{array}$ & $\stackrel{O}{\xi}$ & $\sum^{\infty}$ & ర్ల & $\begin{array}{l}0 \\
\tilde{Z}^{N}\end{array}$ & $\stackrel{0}{\mathscr{V}^{\prime}}$ & $O^{n}$ & 首 & $\omega$ \\
\hline
\end{tabular}




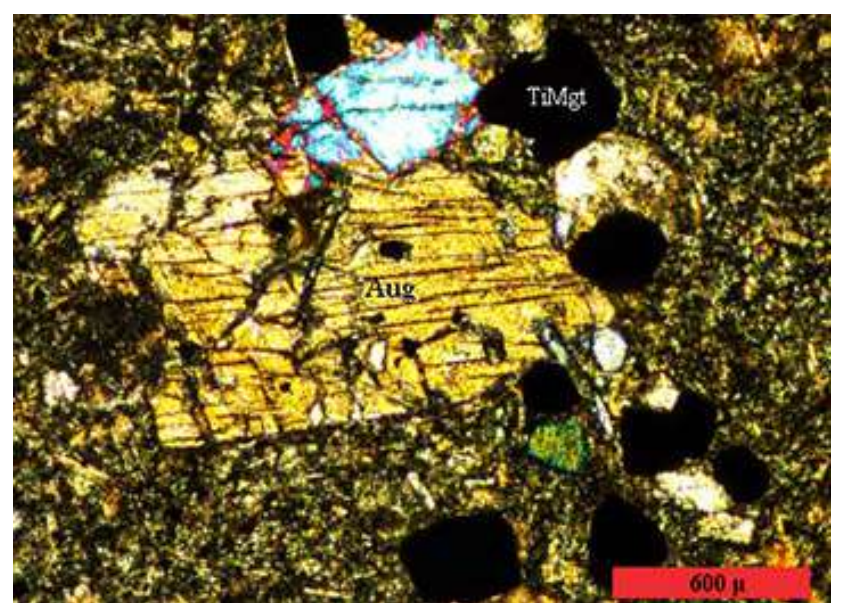

Fig. 2. Intergrowth of titanomagnetite (TiMgt) with highly-calcium augite (Aug) in clinopyroxene basalt. picrites, olivine and chrome spinel as idiomorphic deposits form interrelated grains, whereas chrome spinel in picrobasalt is replaced by chrome titanomagnetite. Labradorite-bytownite plagioclase and high-calcium augite impregnations continue to undergo the process of crystallization.

In the subsequent differentiates, distinctly cut grains of titanomagnetite grow together with high-calcium augite (Fig. 2). In relation to porphyry generations of mineral parageneses, the early stage of crystallization is characterized by olivine-chrome spinel balance. The temperature of crystallization of those impregnations, calculated according to Fabries' thermometer (Fabries, 1979), equaled $1,050-1,100{ }^{\circ} \mathrm{C}$ (Table 2).

Table 2. Chemical compositions of olivines and chrome-spinelides of olivine-chrome-spinel picrites of the Gazakh depression

\begin{tabular}{|c|c|c|c|c|c|c|}
\hline Rocks & \multicolumn{3}{|c|}{ olivine } & \multicolumn{3}{|c|}{ chrome-spinel } \\
\hline sample & 1 & 2 & 3 & 1 & 2 & 3 \\
\hline $\mathrm{SiO}_{2}$ & 39.40 & 38.44 & 39.14 & n.d. & n.d. & n.d. \\
\hline $\mathrm{TiO}_{2}$ & 0.24 & 0.30 & 0.34 & 0.06 & 0.06 & 0.06 \\
\hline $\mathrm{Al}_{2} \mathrm{O}_{3}$ & n.d. & n.d. & n.d. & 14.28 & 14.64 & 12.24 \\
\hline $\mathrm{Cr}_{2} \mathrm{O}_{3}$ & n.d. & n.d. & n.d. & 46.75 & 47.33 & 50.30 \\
\hline $\mathrm{Fe}_{2} \mathrm{O}_{3}$ & 0.37 & 0.37 & 0.42 & 7.36 & 8.36 & 6.38 \\
\hline $\mathrm{FeO}$ & 16.12 & 18.60 & 15.86 & 18.75 & 18.86 & 16.60 \\
\hline $\mathrm{MnO}$ & 0.23 & 0.29 & 0.26 & 0.18 & 0.21 & 0.33 \\
\hline $\mathrm{MgO}$ & 42.38 & 41.58 & 43.86 & 12.36 & 10.15 & 13.64 \\
\hline$\Sigma$ & 98.74 & 99.58 & 99.88 & 99.74 & 99.61 & 99.55 \\
\hline
\end{tabular}

In all types of basalts and dolerites, titanomagnetite is characterized by comparatively high content of titanium and homogenous structure. According to microprobe, chemical (Table 3, 4), X-ray diffraction $(\alpha=8.484-8.516 \AA)$ analyses, the content of ulvospinel molecules ranges $50 \%$ to $64 \%$.

According to the magnetite-ilmenite thermometer, crystallization of porphyry generations of minerals of basalts and dolerites most likely took place in somewhat heightened thermobaric conditions $\left(\mathrm{t}=1030-1100^{\circ} \mathrm{C}\right.$, $\left.\mathrm{fo}_{2}=-9.7-9.2\right)($ Table 3$)$, and the crystallization depth ranges $18-21 \mathrm{~km}$ (Fig. 3).

Presence of inclusions of moderately titanium magnetite in the pyroxene and hornblende phenocrysts that the andesite-basalt melt was crystallized in comparatively more oxidative conditions. Therefore, the depth (4-11 km) (Fig. 3) and temperature $(\mathrm{t}=750$ $850^{\circ} \mathrm{C}$ ) of crystallization respectively decrease.

Phenocrysts of titanomagnetite in basalts and dolerites of the basalt-andesibasalt complex of the Agjakand and Agdara depressions developed in several relatively heightened thermobaric conditions (Table $1)$. Therefore, the calculated Curie points $(\mathrm{Tc}=140$ $250)$ and accordingly the depth of crystallization at the intermediate site increase (20-22 km) (Table 3, Fig. 3).
In the Upper Santonian-lower Campanian periods, change of the previous regime and decrease in permeability of the Earth crust in Agjakand and Agdara depressions hindered the elevation of fractioned portion of the melt. Therefore, highly heated basalt melt has been thermally interacting with above-embedded arkose sandstones, which developed during destruction of the Lower Jurassic quartzitic-plagioporphyry volcanites, causing those sandstones to melt. Thus, from the rhyolite melt that was developing, crust dacites and rhyodacites formed (Table 1).

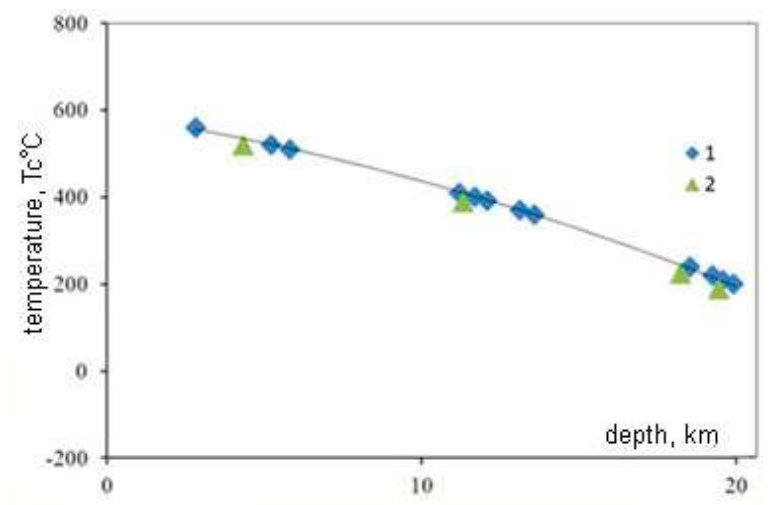

Fig. 3. Dependence of Curie temperature of iron-titanium minerals on depth of Late Cretaceous volcanic complexes of the Gazakh (1) and Agjakand (2) depressions (Kawai, 1956). 


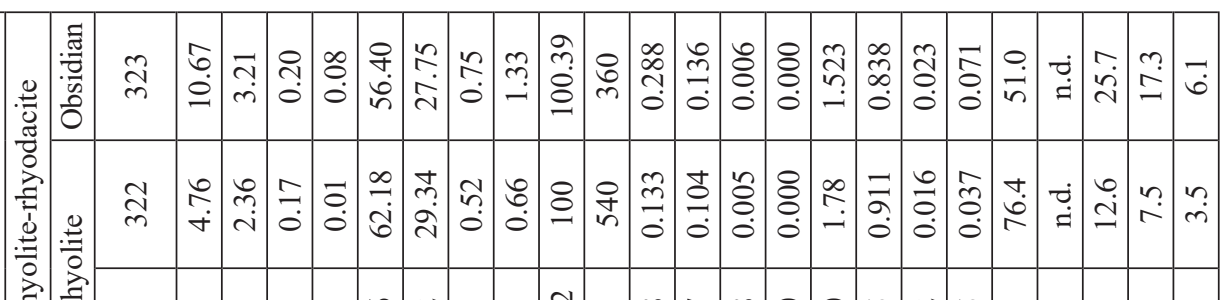

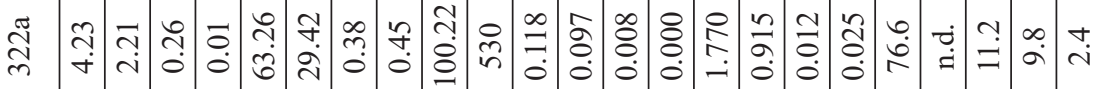

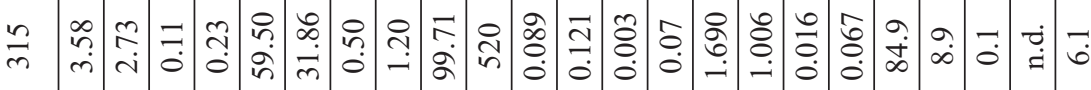

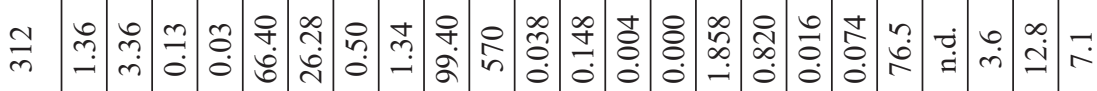

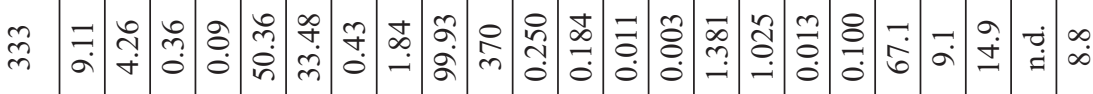

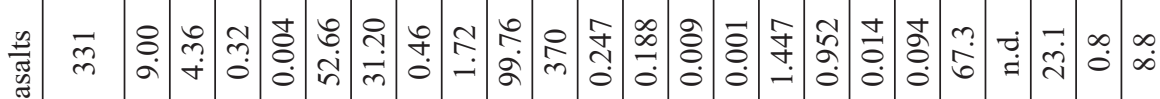

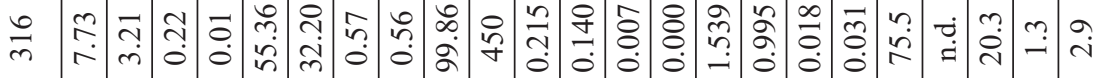

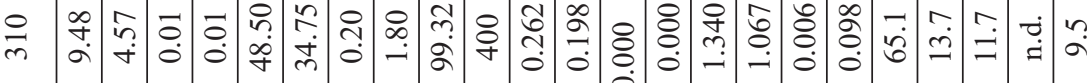

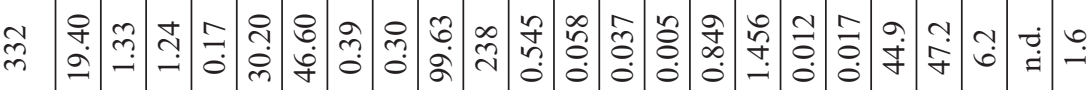

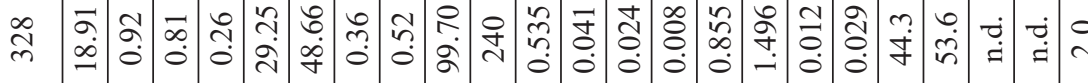

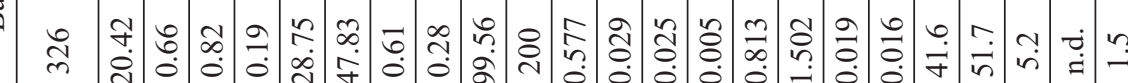

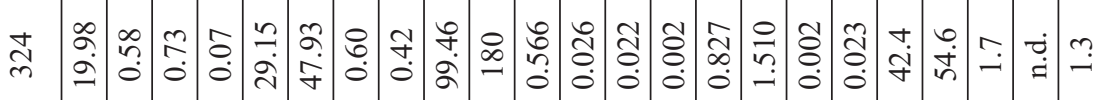

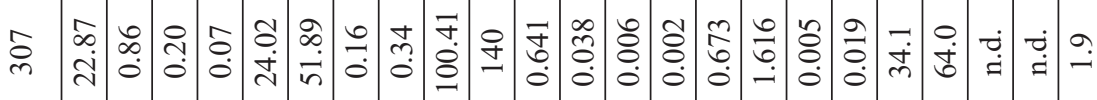

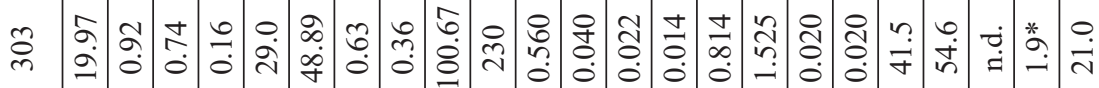

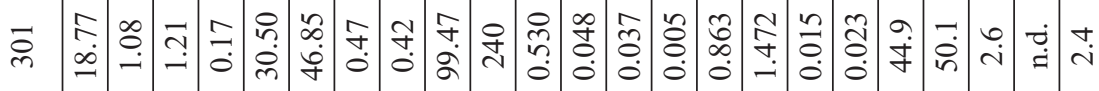

足

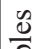


Continuation of Table 3

\begin{tabular}{|c|c|c|c|c|c|c|c|c|c|}
\hline \multicolumn{10}{|c|}{ Agjakand depression } \\
\hline \multirow{3}{*}{$\begin{array}{c}\text { Complexes } \\
\text { Rocks } \\
\text { component } \\
\end{array}$} & \multicolumn{8}{|c|}{ Basalt-andesibasalt } & \multirow{3}{*}{$\begin{array}{c}\text { Rhyolite-rhyodacite } \\
\text { Dacite } \\
338\end{array}$} \\
\hline & \multicolumn{2}{|c|}{ Dolerites } & \multicolumn{4}{|c|}{ Basalts } & \multicolumn{2}{|c|}{ Andesibasalts } & \\
\hline & 359 & 360 & 346 & 350 & 358 & 363 & 334 & 347 & \\
\hline $\mathrm{TiO}_{2}$ & 22.75 & 23.0 & 18.04 & 20.53 & 22.92 & 19.60 & 9.28 & 3.45 & 3.43 \\
\hline $\mathrm{Al}_{2} \mathrm{O}_{3}$ & 2.16 & 2.06 & 1.24 & 1.16 & 1.31 & 1.40 & 1.75 & 4.60 & 3.26 \\
\hline $\mathrm{V}_{2} \mathrm{O}_{3}$ & 0.36 & 0.43 & 0.38 & 0.32 & 0.36 & 0.33 & 0.16 & 0.15 & 0.10 \\
\hline $\mathrm{Cr}_{2} \mathrm{O}_{3}$ & 0.75 & 0.86 & 0.43 & 0.42 & 0.42 & 0.41 & 0.12 & 0.12 & 0.08 \\
\hline $\mathrm{Fe}_{2} \mathrm{O}_{3}$ & 21.69 & 28.80 & 33.29 & 31.93 & 25.23 & 32.10 & 49.03 & 63.80 & 64.25 \\
\hline $\mathrm{FeO}$ & 50.20 & 49.30 & 42.80 & 44.77 & 48.75 & 45.30 & 32.60 & 25.60 & 26.74 \\
\hline $\mathrm{MnO}$ & 1.36 & 1.40 & 3.21 & 0.35 & 0.26 & 0.55 & 0.34 & 0.20 & 0.36 \\
\hline $\mathrm{MgO}$ & 0.64 & 0.52 & 0.43 & 0.52 & 0.43 & 0.48 & 2.52 & 1.82 & 1.31 \\
\hline$\sum$ & 99.91 & 99.99 & 99.82 & 100.2 & 99.68 & 100.17 & 99.40 & 99.74 & 99.53 \\
\hline $\mathrm{Tc}^{\circ} \mathrm{C}$ & 150 & 150 & 160 & 200 & 150 & 240 & 400 & 530 & 530 \\
\hline $\mathrm{Ti}$ & 0.634 & 0.639 & 0.508 & 0.565 & 0.639 & 0.545 & 0.255 & 0.094 & 0.095 \\
\hline $\mathrm{Al}$ & 0.094 & 0.090 & 0.054 & 0.050 & 0.027 & 0.051 & 0.204 & 0.197 & 0.142 \\
\hline $\mathrm{V}$ & 0.011 & 0.013 & 0.011 & 0.015 & 0.011 & 0.010 & 0.005 & 0.004 & 0.003 \\
\hline $\mathrm{Cr}$ & 0.022 & 0.025 & 0.012 & 0.012 & 0.012 & 0.022 & 0.003 & 0.003 & 0.002 \\
\hline $\mathrm{Fe}^{+3}$ & 0.605 & 0.623 & 0.937 & 0.880 & 0.704 & 0.893 & 1.358 & 1.747 & 1.786 \\
\hline $\mathrm{Fe}^{+2}$ & 1.556 & 1.523 & 1.339 & 1.393 & 1.512 & 1.401 & 0.991 & 0.719 & 0.826 \\
\hline $\mathrm{Mn}$ & 0.042 & 0.044 & 0.102 & 0.011 & 0.008 & 0.017 & 0.010 & 0.006 & 0.011 \\
\hline $\mathrm{Mg}$ & 0.035 & 0.029 & 0.024 & 0.028 & 0.024 & 0.026 & 0.137 & 0.099 & 0.072 \\
\hline Mgt & 33.1 & 34.2 & 47.6 & 43.5 & 35.7 & 43.9 & 65.8 & 75.5 & 80.0 \\
\hline Usp & 63.4 & 57.2 & 44.3 & 37.1 & 49.7 & 41.2 & 9.6 & n.d. & n.d. \\
\hline Il & n.d. & 5.8 & 5.7 & 17 & 12.13 & 11.9 & 14.8 & 8.1 & 5.1 \\
\hline $\mathrm{Hm}$ & n.d. & n.d. & n.d. & n.d. & n.d. & n.d. & n.d. & 7.9 & 8.5 \\
\hline $\mathrm{Sp}$ & 3.5 & 2.8 & 2.4 & 2.4 & 2.3 & 3.0 & 9.8 & 8.5 & 6.3 \\
\hline
\end{tabular}

Indexes of minerals: Mgt-magnetites; Usp - ulvospinel; Il-ilmenite; $\mathrm{Sp}$ - spinel; Hm - hematite; Tc - calculated value of Curie points

Table 4. Chemical, crystal-chemical and mineral compositions of ilmenites of the Gazakh and Agjakand depressions

\begin{tabular}{|c|c|c|c|c|c|c|c|c|c|c|c|c|}
\hline \multicolumn{8}{|c|}{ Gazakh depression } & \multicolumn{5}{|c|}{ Agjakand depression } \\
\hline Complexes & \multicolumn{12}{|c|}{ Basalt-andesibasalts } \\
\hline $\begin{array}{l}\text { № samples } \\
\text { components }\end{array}$ & 300 & 307 & 324 & 328 & 310 & 315 & 322 & $\begin{array}{c}\text { No samples } \\
\text { component }\end{array}$ & 359 & 360 & 346 & 334 \\
\hline $\mathrm{TiO}_{2}$ & 48.66 & 47.2 & 48.64 & 48.38 & 49.16 & 48.3 & 46.63 & $\mathrm{TiO}_{2}$ & 49.16 & 48.60 & 47.63 & 48.50 \\
\hline $\mathrm{Al}_{2} \mathrm{O}_{3}$ & 0.64 & 0.93 & 0.34 & 0.16 & 0.75 & 1.15 & 1.34 & $\mathrm{Al}_{2} \mathrm{O}_{3}$ & 0.36 & 0.23 & 0.36 & 1.18 \\
\hline $\mathrm{V}_{2} \mathrm{O}_{3}$ & 0.28 & 0.2 & 0.27 & 0.38 & 0.18 & 0.04 & 0.21 & $\mathrm{~V}_{2} \mathrm{O}_{3}$ & 0.28 & 0.25 & 0.66 & 0.42 \\
\hline $\mathrm{Cr}_{2} \mathrm{O}_{3}$ & 0.26 & 0.18 & 0.1 & 0.16 & 0.34 & 0.09 & 0.14 & $\mathrm{Cr}_{2} \mathrm{O}_{3}$ & 0.18 & 0.19 & 0.16 & 0.04 \\
\hline $\mathrm{Fe}_{2} \mathrm{O}_{3}$ & 6.5 & 9.17 & 7.42 & 7.52 & 5.2 & 6.8 & 9.68 & $\mathrm{Fe}_{2} \mathrm{O}_{3}$ & 6.21 & 6.98 & 9.25 & 6.53 \\
\hline $\mathrm{FeO}$ & 42.99 & 40.11 & 42.13 & 41.93 & 42.2 & 41.74 & 41.09 & $\mathrm{FeO}$ & 42.72 & 41.97 & 40.78 & 41.38 \\
\hline $\mathrm{MnO}$ & 0.56 & 0.94 & 0.66 & 0.58 & 1.36 & 1.18 & 0.78 & $\mathrm{MnO}$ & 1.34 & 1.60 & 1.36 & 1.58 \\
\hline Mgo & 0.24 & 0.73 & 0.54 & 0.66 & 0.34 & 0.36 & 0.23 & $\mathrm{MgO}$ & 0.06 & 0.07 & 0.34 & 0.36 \\
\hline$\sum$ & 100.13 & 99.46 & \begin{tabular}{|l|}
100.1 \\
\end{tabular} & 99.77 & 99.53 & 99.66 & 100.1 & $\sum$ & 100.31 & 99.89 & 100.54 & 99.99 \\
\hline $\mathrm{t}^{\circ} \mathrm{C}$ & 1030 & 1080 & 1050 & 1100 & 850 & 750 & 760 & $t^{\circ} \mathrm{C}$ & 1120 & 1100 & 1150 & 780 \\
\hline $\operatorname{lgfo}_{2}$ & -9.7 & -9.8 & -10 & -9.2 & -12.5 & -11 & -12.5 & $\operatorname{lgfo}_{2}$ & -9.8 & -9.6 & -8.6 & -12.5 \\
\hline $\mathrm{Ti}$ & 0.922 & 0.896 & 0.921 & \begin{tabular}{|l|}
0.919 \\
\end{tabular} & 0.934 & 0.916 & 0.882 & $\mathrm{Ti}$ & 0.931 & 0.926 & 0.899 & 0.916 \\
\hline $\mathrm{Al}$ & 0.019 & 0.028 & \begin{tabular}{|l|}
0.01 \\
\end{tabular} & 0.005 & 0.022 & 0.034 & 0.039 & $\mathrm{Al}$ & 0.011 & 0.007 & 0.011 & 0.035 \\
\hline V & 0.006 & 0.004 & 0.005 & \begin{tabular}{|l|}
0.008 \\
\end{tabular} & 0.004 & 0.001 & 0.004 & $\mathrm{~V}$ & 0.006 & 0.005 & 0.013 & 0.008 \\
\hline $\mathrm{Cr}$ & 0.005 & \begin{tabular}{|l|}
0.003 \\
\end{tabular} & 0.002 & \begin{tabular}{|l|}
0.003 \\
\end{tabular} & 0.007 & \begin{tabular}{|l|l}
0.002 \\
\end{tabular} & 0.003 & $\mathrm{Cr}$ & 0.003 & 0.004 & 0.004 & 0.001 \\
\hline $\mathrm{Fe}^{+3}$ & 0.123 & \begin{tabular}{|l|l|}
0.174 \\
\end{tabular} & \begin{tabular}{|l|}
0.141 \\
\end{tabular} & 0.143 & \begin{tabular}{|l}
0.099 \\
\end{tabular} & \begin{tabular}{|l|l|}
0.129 \\
\end{tabular} & 0.183 & $\mathrm{Fe}^{+3}$ & 0.118 & 0.133 & 0.175 & 0.123 \\
\hline $\mathrm{Fe}^{+2}$ & 0.904 & 0.847 & 0.887 & 0.885 & 0.892 & 0.88 & 0.865 & $\mathrm{Fe}^{+2}$ & 0.900 & 0.889 & 0.857 & 0.869 \\
\hline $\mathrm{Mn}$ & 0.012 & 0.02 & 0.014 & \begin{tabular}{|l|}
0.012 \\
\end{tabular} & 0.029 & 0.025 & 0.016 & $\mathrm{Mn}$ & 0.029 & 0.034 & 0.029 & 0.034 \\
\hline $\mathrm{Mg}$ & 0.009 & 0.028 & \begin{tabular}{|l|}
0.02 \\
\end{tabular} & 0.025 & 0.013 & 0.013 & 0.008 & $\mathrm{Mg}$ & 0.002 & 0.003 & 0.013 & 0.013 \\
\hline $\mathrm{MnTiO}_{3}$ & 1.1 & 2.00 & 1.9 & 2.3 & 2.7 & 2.3 & 1.42 & $\mathrm{MnTiO}_{3}$ & 2.7 & 3.2 & 2.6 & 3.2 \\
\hline $\mathrm{MgTiO}_{3}$ & 0.8 & 2.8 & 1.3 & 1 & 1.2 & 1.2 & 0.7 & $\mathrm{MgTiO}_{3}$ & 0.2 & 0.3 & 1.2 & 1.2 \\
\hline $\mathrm{FeTiO}_{3}$ & 83.6 & 84.7 & 82.2 & 81.7 & 83.7 & 81 & 76.4 & $\mathrm{FeTiO}_{3}$ & 84.2 & 82.7 & 77.8 & 80.1 \\
\hline $\mathrm{Fe}_{2} \mathrm{O}_{3}$ & 14.5 & 10.5 & 14.6 & 14.9 & 12.7 & 15.5 & 21.2 & $\mathrm{Fe}_{2} \mathrm{O}_{3}$ & 12.9 & 13.8 & 18.4 & 15.5 \\
\hline
\end{tabular}

Indexes of minerals: $\mathrm{FeTiO}_{3}$-ilmenite, $\mathrm{MgTiO}_{3}$ - geikielite, $\mathrm{MnTiO}_{3}$ - pyrophanite. 
The latter are different from the similar dacites and rhyodacites of the Gazakh depression because of the somewhat increased content of large-ion alkaline (K, $\mathrm{Rb})$ and alkaline-earth (Ba) elements, discreetness, and also presence of arkose sandstones in the distribution range of those rocks.

Based on the analyses of the presented materials, we may conclude that in the compound of picrobasalts and picrodolerites, in relatively more high-baric conditions, crystallization was undergone by olivinechrome spinel paragenesis $\left(\mathrm{t}=1,080-1,100^{\circ} \mathrm{C}\right)$. In the composition of the subsequent differentiates, over the process of evolution of the olivine-basalt melt, due to crystallization differentiation, chrome spinel has been excluded from the paragenesis and therefore the content of olivine partly decreases. The following paragenesis is accompanied by bytownite plagioclase, augite clinopyroxene, chrome-impoverished titanomagnetite. Increase in the content of ulvospinel molecules and decrease in the calculated and experimental Curie points $\left(150-240{ }^{\circ} \mathrm{C}\right)$ indicate that titanomagnetites in basalts and dolerites of the Agjakand and Agdara depressions, compared with the similar petrographic types of rocks of the Gazakh depression, have crystallized in high thermobaric conditions (Table 3, Fig. 4).

Due to palligenic transformation of partly hydrated arkose sandstones in dacites and rhyodacites, in the oxidative setting, crystallization of hematite, goethite and maghemite occurred.

The Goycha-Akeri structural-formation zone (the Khojavand and Azykh depressions), starting from the eastern bank of Goycha Lake, can be seen in the southeast direction to the Araz River and is located between the Lok-Garabagh and Miskhan-Kafan structural-formation zones.

Iron-titanium oxide minerals in the composition of the rocks of Late Cretaceous formations of tephrite- teshenite and trachybasalt-trachydolerite complexes of Khojavand, and also trachybasalt-trachyandesite complex of the Azykh depressions of the Goycha-Akeri structural-formation zone of the Lesser Caucasus are represented by moderately titanium and high alumina titanomagnetite, ilmenite, maghemite, hematite and others.

Geological structure and also petrologicalmineralogic peculiarities of the trachybasalttrachydolerite and tephrite-teshenite complexes of the Khojavand depression were analyzed in previous articles (Akhundov, 2003, Avdeev i dr, 1989, Mamedov i dr., 2012, 2013, 2015).

The trachybasalt-trachydolerite complex that characterizes the early substage of the Santonian volcanism is composed of olivine, clinopyroxeneplagioclase and plagioclase trachybasalts and trachydolerites (Table 1).

The rocks of tephrite-teshenite complex correspond to the late substage of the Santonian volcanism. The effusive-pyroclastic facies consist of picrotephrite, melano- and leukotephrite. The mentioned rocks often contain several rolled inclusions of subalkaline picrite, and also singular large megacrystals of clinopyroxene and phlogopite plates $(0.5 \times 2.5 \mathrm{~cm})$. As inclusions, chrome spinel and titanomagnetite are in most cases confined to clinopyroxene phenocrysts. This indicates that the process of crystallization of tephrite melt at the intermediate site occurred in the oxidative setting. Therefore, crystallization of the main plagioclase was hindered, leading to enrichment of titanomagnetite with specifically spinel, and clinopyroxene - with calcium tschermakite molecules.

Early stage of crystallization of the subalkaline picrite melt took place according to olivine-chrome spinel Fabries' geothermometer (Fabries, 1979) in the temperature of $980-1,100{ }^{\circ} \mathrm{C}$ (Table. 5,6$)$.

Table 5. Chemical, crystal-chemical and minal compositions of chrome-spinels and titanomagnetite rocks of tephrite-teshenite, trachybasalt-trachydolerite complexes of the Khojavand depression.

\begin{tabular}{|c|c|c|c|c|c|c|c|c|c|c|c|c|c|}
\hline Complex & \multicolumn{8}{|c|}{ Tephrite-teshenite } & \multicolumn{5}{|c|}{ Trachybasalt-trachydolerite } \\
\hline Components & $372 v$ & $372 \mathrm{a}$ & $372 b$ & 376 & 375 & 381 & 399 & $399 a$ & 379 & 380 & 388 & 392 & 370 \\
\hline $\mathrm{TiO}_{2}$ & 2.12 & 3.47 & 2.93 & 9.79 & 2.6 & 10.93 & 16.59 & 18.2 & 10.2 & 9.36 & 9.16 & 0.81 & 12.14 \\
\hline $\mathrm{Al}_{2} \mathrm{O}_{3}$ & 18.05 & 12.38 & 13.87 & 10.99 & 16.82 & 3.24 & 2.07 & 1.46 & 4.6 & 3.31 & 5.38 & 0.59 & 4.89 \\
\hline $\mathrm{V}_{2} \mathrm{O}_{3}$ & 0.15 & 0.23 & 0.23 & n.d. & 0.26 & n.d. & n.d. & n.d. & n.d. & n.d. & n.d. & n.d. & n.d. \\
\hline $\mathrm{Cr}_{2} \mathrm{O}_{3}$ & 33.50 & 34.13 & 31.40 & 13.04 & 34.42 & 0.01 & 0.09 & 0.1 & 1.19 & 0.01 & 0.01 & n.d. & 0.23 \\
\hline $\mathrm{Fe}_{2} \mathrm{O}_{3}$ & 16.80 & 16.31 & 17.68 & 25.83 & 14.47 & 44.66 & 34.51 & 32.24 & 43.68 & 48.00 & 47.43 & 67.09 & 41.4 \\
\hline $\mathrm{FeO}$ & 14.47 & 23.85 & 25.99 & 31.02 & 16.31 & 38.81 & 44.37 & 46.8 & 39.05 & 37.24 & 33.44 & 21.36 & 36.43 \\
\hline $\mathrm{NiO}$ & 0.88 & 0.12 & 0.12 & n.d. & 0.78 & n.d. & n.d. & n.d. & n.d. & n.d. & n.d. & n.d. & n.d. \\
\hline $\mathrm{MnO}$ & 0.24 & 0.14 & 0.53 & 0.36 & 0.28 & 0.54 & 0.98 & 0.68 & 0.04 & 0.03 & 0.04 & 0.31 & 0.61 \\
\hline $\mathrm{MgO}$ & 13.71 & 8.59 & 7.24 & 8.44 & 12.6 & 1.5 & 0.84 & 0.46 & 1.52 & 1.96 & 4.56 & 0.21 & 3.91 \\
\hline$\sum$ & 99.92 & 99.22 & 99.99 & 99.47 & 98.54 & 99.69 & 99.45 & 99.94 & 100.3 & 99.91 & 100 & 90.37 & 99.61 \\
\hline $\mathrm{Tc}^{\circ} \mathrm{C}$ & n.d. & n.d. & n.d. & n.d. & n.d. & 360 & 250 & 230 & 377 & 395 & 380 & 350 & 360 \\
\hline $\mathrm{t},{ }^{\circ} \mathrm{C}$ & 1040 & 1200 & 1150 & 1180 & 995 & 980 & 1020 & 1030 & 758 & n.d. & n.d. & n.d. & 960 \\
\hline $\operatorname{lgfo}_{2}$ & -5.90 & -7.2 & -5.39 & -6.32 & -5.68 & -10 & -11 & -10 & $-14,2$ & n.d. & n.d. & n.d. & $-10,2$ \\
\hline
\end{tabular}


Continuation of Table 5

\begin{tabular}{|c|c|c|c|c|c|c|c|c|c|c|c|c|c|}
\hline Complex & \multicolumn{8}{|c|}{ Tephrite-teshenite } & \multicolumn{5}{|c|}{ Trachybasalt-trachydolerite } \\
\hline $\begin{array}{l}\text { № samples } \\
\text { Components }\end{array}$ & $372 \mathrm{v}$ & $372 \mathrm{a}$ & $372 b$ & 376 & 375 & 381 & 399 & $399 \mathrm{a}$ & 379 & 380 & 388 & 392 & 370 \\
\hline $\mathrm{Ti}$ & 0.050 & 0.087 & 0.073 & 0.248 & 0.062 & 0.305 & 0.467 & 0.51 & 0.281 & 0.26 & 0.247 & 0.023 & 0.33 \\
\hline $\mathrm{Al}$ & 0.669 & 0.487 & 0.547 & 0.437 & 0.634 & 0.142 & 0.091 & 0.064 & 0.199 & 0.144 & 0.227 & 0.027 & 0.208 \\
\hline $\mathrm{V}$ & 0.004 & 0.006 & 0.006 & 0.000 & 0.006 & 0.000 & 0.003 & 0.003 & 0.000 & 0.000 & 0.000 & 0.000 & 0.000 \\
\hline $\mathrm{Cr}$ & 0.833 & 0.913 & 0.831 & 0.348 & 0.870 & 0.000 & 0.000 & 0.000 & 0.034 & 0.001 & 0.002 & 0.000 & 0.007 \\
\hline $\mathrm{Fe}^{+3}$ & 0.398 & 0.410 & 0.445 & 0.718 & 0.348 & 1.248 & 0.972 & 0.908 & 1.204 & 1.335 & 1.279 & 1.927 & 1.126 \\
\hline $\mathrm{Fe}^{+2}$ & 0.380 & 0.665 & 0.727 & 0.813 & 0.436 & 1.205 & 1.389 & 1.465 & 1.197 & 1.151 & 1.002 & 1.001 & 1.1 \\
\hline $\mathrm{Mn}$ & 0.006 & 0.004 & 0.003 & 0.010 & 0.007 & 0.002 & 0.031 & 0.022 & 0.001 & 0.001 & 0.001 & 0.01 & 0.018 \\
\hline $\mathrm{Mg}$ & 0.642 & 0.428 & 0.361 & 0.424 & 0.601 & 0.083 & 0.047 & 0.026 & 0.083 & 0.108 & 0.243 & 0.012 & 0.21 \\
\hline $\mathrm{Ni}$ & 0.022 & 0.004 & 0.003 & 0.000 & 0.020 & 0.000 & 0.000 & 0.000 & 0.000 & 0.000 & 0.000 & 0.000 & 0.000 \\
\hline $\mathrm{Sp}$ & 29.4 & 24.0 & 23.2 & 21.1 & 27.0 & & & & & & & & \\
\hline $\mathrm{MgCr}_{2} \mathrm{O}_{4}$ & 32.2 & 18.1 & 7.4 & 17.0 & 24.2 & & & & & & & & \\
\hline $\mathrm{FeCr}_{2} \mathrm{O}_{4}$ & 7.6 & 26.3 & 27.8 & - & 12.9 & & & & & & & & \\
\hline Mgt & 21.3 & $22.9^{*}$ & $31.6^{*}$ & 37.8 & $30.6^{*}$ & & & & & & & & \\
\hline Usp & 9.6 & 8.6 & 10.0 & 24.1 & 5.31 & & & & & & & & \\
\hline $\mathrm{Sp}$ & & & & & & 7.1 & 4.6 & 2.6 & 6.7 & 7.2 & 11.2 & 1.2 & 10.4 \\
\hline Mgt & & & & & & 62.3 & 48.7 & 46.2 & 70.7 & 66.8 & 63.8 & 96.5 & 56.6 \\
\hline Usp & & & & & & 30.5 & 46.7 & 51.2 & 3.0 & 25.9 & 24.4 & 2.3 & 33.0 \\
\hline Il & & & & & & n.d. & n.d. & n.d. & 19.6 & 0.1 & 0.5 & n.d. & n.d. \\
\hline
\end{tabular}

*- Wüstite is involved in the subordinate amount.

$372 \mathrm{v}$ - subalkaline picrite, $372 \mathrm{a}$ - subalkaline picrotephrite, $372 \mathrm{~b}, 376$ - melano-tephrite, $375-$ megacrystal of chrome spinel, 381 - leucocratic tephrite, 399 - melano-teshenite, 399a-leucoteshenite,
379, 380 - analcime-clinopyroxene trachybasalt, 388 - clinopyroxene trachybasalt, 392 - plagioclase trachybasalt, 370 - trachydolerite.

Table 6. Chemical compositions of olivine of rocks of tephrite-teshenite complex of the Khojavand depression

\begin{tabular}{|l|c|c|c|}
\hline components & $372 \mathrm{a}$ & $372 \mathrm{~b}$ & $382 \mathrm{~b}$ \\
\hline $\mathrm{SiO}_{2}$ & 38.30 & 37.20 & 38.20 \\
\hline $\mathrm{FeO}$ & 21.36 & 25.70 & 20.40 \\
\hline $\mathrm{MgO}$ & 40.22 & 36.80 & 41.70 \\
\hline$\sum$ & 99.88 & 99.70 & 100.30 \\
\hline
\end{tabular}

In the composition of melano- and leucocratic tephrites, the share of phenocrysts of olivine, chromediopside, chrome spinel gradually decreases, whereas in the phenocrysts, the content of fayalite, ferrosilite and ulvospinel minerals notably increases. This indicates that melano- and leucocratic tephrites are differentiates of sub-alkaline picrite melt.

In the composition of tephrites, there is a small amount of phenocrysts of kaersutite-barkevikite amphibole, and mainly the late magmatic analcimes, indicating oxidative conditions of crystallization. Therefore, crystallization of the main plagioclase was slowed, and therefore a certain share of alumina dissolves in the compound of moderately titanium titanomagnetite, causing the latter to crystallize before the clinopyroxene impregnations. This is indicated by the presence of inclusions of distinctly cut depressions of titanomagnetite in clinopyroxene (Fig. 4). Therefore the temperature of crystallization of porphyry generations of the minerals of tephrite melt somewhat decreases (760$820^{\circ} \mathrm{C}$ ), while the partial pressure of oxygen increases $\left(\operatorname{lgfo}_{2}=-9 \cdot 2-10\right)$. According to the calculated (Table 5, 7) and experimental values of Curie points, the depth of crystallization of tephrite melt at intermediate site somewhat decreases (6-12 km) (Fig. 5). 
Table 7. Chemical, crystal-chemical and minal composition of ilmenites of tephrite-teshenite and trachybasalt-trachydolerite complexes of the Khojavand depression

\begin{tabular}{|c|c|c|c|c|c|}
\hline Complexes & \multicolumn{3}{|c|}{ tephrite-teshenite } & \multicolumn{2}{|c|}{ trachybasalt-trachydolerite } \\
\hline $\begin{array}{ll}\text { components } & \text { № samples } \\
\end{array}$ & 381 & 399 & $399 a$ & 370 & 379 \\
\hline $\mathrm{TiO}_{2}$ & 46.74 & 48.31 & 48.36 & 47.68 & 48.50 \\
\hline $\mathrm{Al}_{2} \mathrm{O}_{3}$ & 0.75 & 0.23 & 0.32 & 0.52 & 0.56 \\
\hline $\mathrm{V}_{2} \mathrm{O}_{3}$ & 0.28 & n.d. & n.d. & n.d. & n.d. \\
\hline $\mathrm{Cr}_{2} \mathrm{O}_{3}$ & 0.36 & n.d. & n.d. & 0.48 & 0.36 \\
\hline $\mathrm{Fe}_{2} \mathrm{O}_{3}$ & 10.05 & 7.39 & 7.67 & 8.06 & 4.06 \\
\hline $\mathrm{FeO}$ & 40.81 & 42.19 & 42.06 & 42.06 & 42.66 \\
\hline $\mathrm{MnO}$ & 0.58 & 0.56 & 0.75 & 0.58 & 0.63 \\
\hline $\mathrm{MgO}$ & 0.42 & 0.66 & 0.38 & 0.26 & 0.33 \\
\hline$\sum$ & 99.99 & 99.63 & 99.54 & 99.64 & 97.1 \\
\hline $\mathrm{Ti}$ & 0.886 & 0.924 & 0.922 & 0.891 & 0.924 \\
\hline $\mathrm{Al}$ & 0.022 & 0.007 & 0.009 & 0.016 & 0.016 \\
\hline $\mathrm{V}$ & 0.006 & 0.000 & 0.000 & 0.000 & 0.000 \\
\hline $\mathrm{Cr}$ & 0.007 & 0.000 & 0.000 & 0.010 & 0.007 \\
\hline $\mathrm{Fe}^{+3}$ & 0.191 & 0.141 & 0.146 & 0.186 & 0.123 \\
\hline $\mathrm{Fe}^{+2}$ & 0.860 & 0.892 & 0.892 & 0.839 & 0.904 \\
\hline $\mathrm{Mn}$ & 0.012 & 0.012 & 0.016 & 0.055 & 0.013 \\
\hline $\mathrm{Mg}$ & 0.016 & 0.025 & 0.014 & 0.001 & 0.012 \\
\hline $\mathrm{MnTiO}_{3}$ & 1.1 & 1.1 & 1.5 & 1.1 & 1.1 \\
\hline $\mathrm{MgTiO}_{3}$ & 1.4 & 2.3 & 1.3 & 0.9 & 1.2 \\
\hline $\mathrm{FeTiO}_{3}$ & 77 & 82.2 & 82.8 & 81.3 & 83.6 \\
\hline $\mathrm{Fe}_{2} \mathrm{O}_{3}$ & 20.5 & 14.4 & 14.4 & 16.7 & 14.1 \\
\hline
\end{tabular}

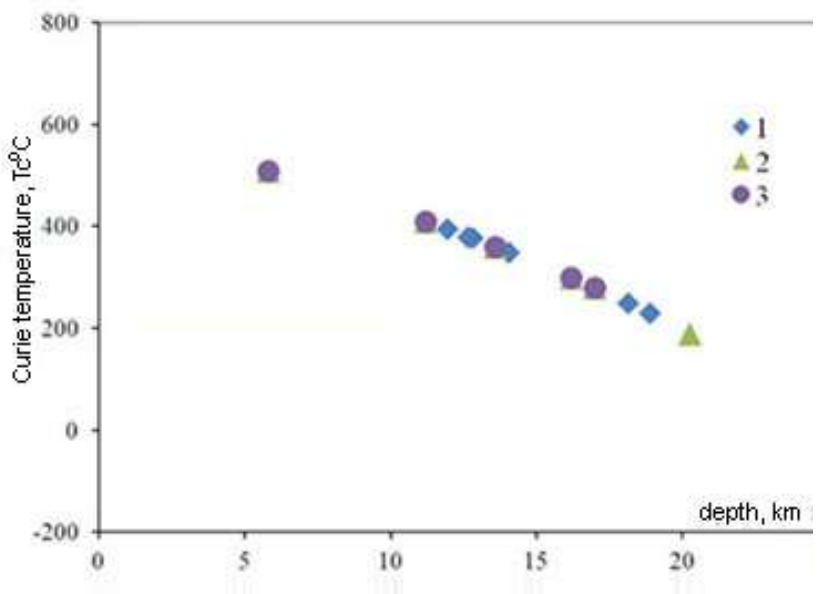

Fig. 4. Inclusions of moderately titanium and alumina titanomagnetites (TiMgt) in salite pyroxene (Sal).

Teshenites, present as thin plate injection, which are vertically composed of picroteshenite, melano-, meso- and leucocratic teshenites, teshenite-syenite, are to a sufficient degree differentiated. Those differentiates are cut by light grey and whitish grey veins of analcime syenites.

Iron-titan oxide minerals in the rocks of the complex of the Azykh depression of the Goycha-Akeri zone are represented by moderately and highly titanous magnetite and singular ilmenites (Tables 8,9).

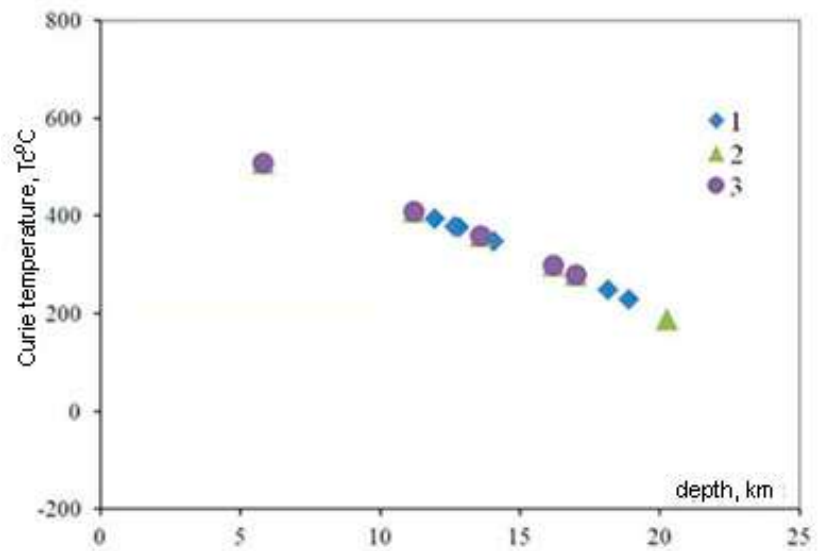

Fig. 5. Dependence of the temperature of Curie ferromagnetic minerals on the depth of Late Cretaceous volcanic complexes of Khojavand (1), Azykh (2) and Gochas (3) depressions of the Lesser Caucasus (Kawai, 1956).

In the rocks of basalt-andesibasalt complex, moderately titanium magnetites crystallized almost at the same time with the clinopyroxene phenocrysts (Table 8, samp. 1, 2). In a number of cases, they are concentrated around amphibole as drops. Along with them, there are also seen shapeless, secondary maghemite and bloody-red hematite. Content of ulvospinel component varies $28 \%$ to $33 \%$.

In the rocks of subalkaline of trachybasalttrachyandesibasalt complex, the content of titan in the titanomagnetite significantly increases (Table 8, 
samp. 3, 4). Unlike the rocks of basalt-andesibasalt complex, titanomagnetite in the rocks of this complex is present as inclusions in clinopyroxenes, which leads to the conclusion that titanomagnetite crystallizes before clinopyroxene.

All determined peculiarities of titanomagnetites indicate development of their impregnations within the differentiates of basalt-andesibasalt complex in moderately thermobaric conditions. However, comparatively earlier crystallization of the phenocrysts of titanomagnetite within the rocks of subalkaline of trachybasalt-trachyandesibasalt complex suggests that the development process took place in relatively deeper and oxidative conditions.

The Miskhan-Kafan structural-formation zone. This structural-formation zone, located in the northeast outskirts of the Goycha-Garabagh zone, is limited by the Lachin-Bashlybel faults in the northwest, and the Girratagh fault in the east.

Structurally, the Gochas depression is confined to the Miskhan-Kafan structural-formation zone. The Late Cretaceous volcanites comprise differentiates of the volcanic complexes confined to the axis zone of this depression.

Unlike the Azykh depression, iron-titan oxide minerals in the rocks of the Gochas depression consist of chrome spinel, picotite, titanomagnetite, maghemite, ilmenite and hematite.

Late Cretaceous volcanic complexes of the Gochas depression fill the narrow long flat-bed structure that strikes northwest. Most scientists (Ostroumova et al.., 1988, Karjakin, 1989) consider that the volcanism and accumulation of sediments came about in the Late Senonian period.

There, similarly to the Azykh depression, the rocks of the basalt-andesibasalt complex were developing during the early substage of volcanism in the Late Senonian period. They are composed of highly titanium olivine-plagioclases, olivine-plagioclase-pyroxene basalts (Table 1). In those poorly differentiated basalts, titanomagnetite in many phenocrysts is involved in singular grains, and is somewhat enriched with titanium (Table 8, samp. 4, 5). Moreover, melanobasalts were determined to contain picotite (Ostroumova et al., 1988). The rocks of trachybasalt-trachyandesibasalt complex, which characterize the late substage of Late Senonian volcanism, are composed of subalkaline trachybasalts, trachyandesibasalts, even trachyandesibasalts, which contain magnetite that is relatively more enriched with titanium (Table 1).

In the most high-temperature conditions, there occurred crystallization of olivine-pyroxene-plagioclase basalts $\left(1,050{ }^{\circ} \mathrm{C}\right)$, while for olivine-plagioclase varieties of basalts, the temperature was calculated as $820-920^{\circ} \mathrm{C}$ (Table 7). The temperature of chrome spinel-olivine equilibrium $\left(\mathrm{SiO}_{2}=39.16, \mathrm{FeO}=22.30\right.$, $\left.\mathrm{MgO}=38.43 ; \mathrm{d}_{\mathrm{hk1} 130}=2.780-2.784 \AA ; \mathrm{Fo}_{73-76 \%}\right)$, present in melanobasalts, was calculated as $1,100-1,150{ }^{\circ} \mathrm{C}$. Presence of homegenity, and also high content of ulvospinel molecule involved in the composition of titanomagnetites indicates that porphyry-like phenocrysts of olivine dolerites and basalts crystallized at the deepest intermediate sites (14-19 km) (Fig. 5).

Table 8. Chemical, crystal-chemical and minal analyses of magnetites of basalt-andesibasalt and trachybasalt-trachyandesibasalt complexes of the Gochas and Azykh depressions.

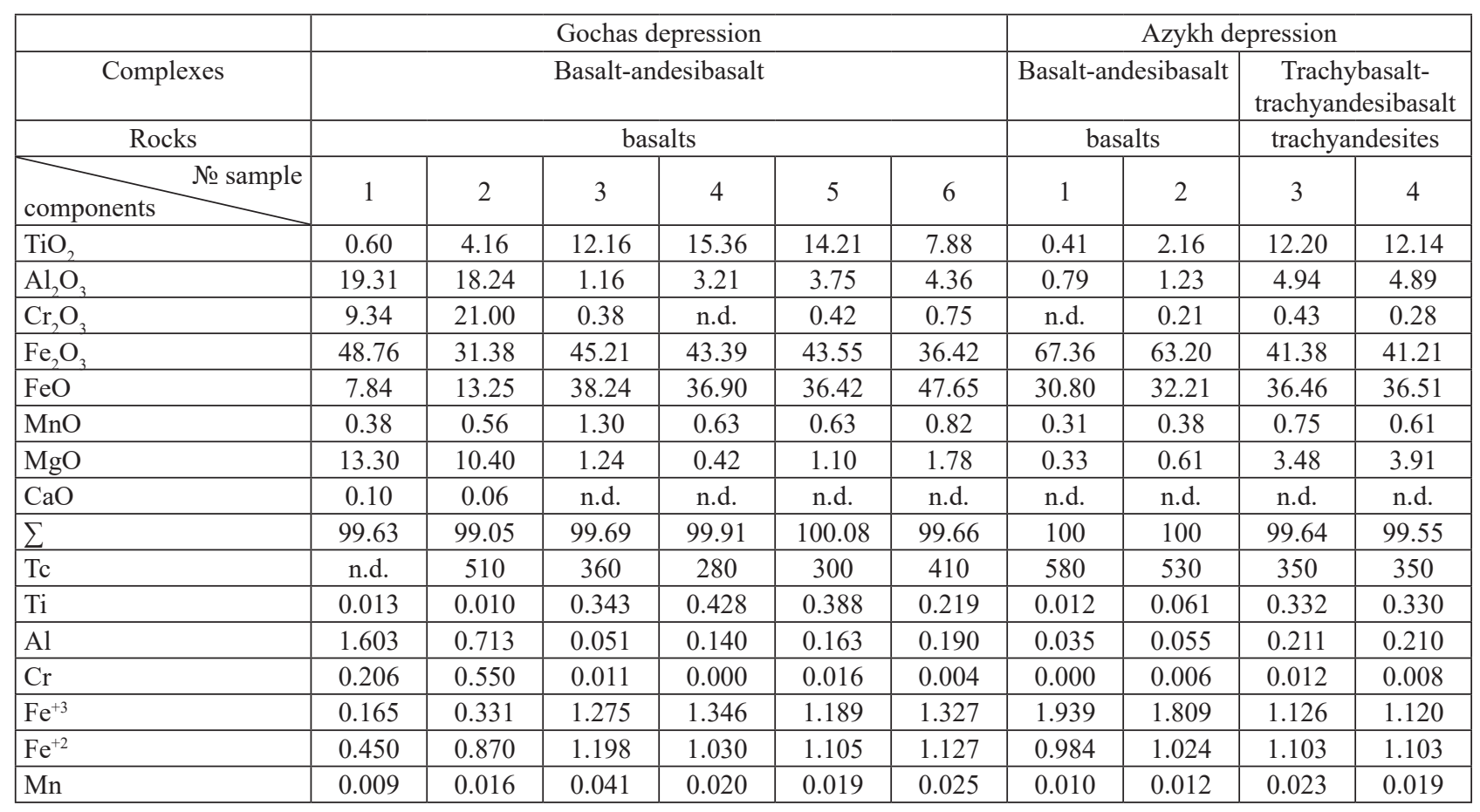


Continuation of Table 5

\begin{tabular}{|c|c|c|c|c|c|c|c|c|c|c|}
\hline \multirow{4}{*}{$\begin{array}{l}\text { Complexes } \\
\text { Rocks } \\
\text { № sample }\end{array}$} & \multirow{2}{*}{\multicolumn{6}{|c|}{$\begin{array}{l}\text { Gochas depression } \\
\text { Basalt-andesibasalt }\end{array}$}} & \multicolumn{4}{|c|}{ Azykh depression } \\
\hline & & & & & & & Basalt-a & esibasalt & $\begin{array}{r}\text { Trach } \\
\text { trachya }\end{array}$ & $\begin{array}{l}\text { asalt- } \\
\text { sibasalt }\end{array}$ \\
\hline & \multicolumn{6}{|c|}{ basalts } & \multicolumn{2}{|c|}{ basalts } & \multicolumn{2}{|c|}{ trachyandesites } \\
\hline & 1 & 2 & 3 & 4 & 5 & 6 & 1 & 2 & 3 & 4 \\
\hline $\mathrm{Mg}$ & 0.553 & 0.514 & 0.069 & 0.023 & 0.059 & 0.098 & 0.019 & 0.034 & 0.186 & 0.211 \\
\hline $\mathrm{Ca}$ & 0.003 & 0.002 & n.d. & n.d. & n.d. & n.d. & n.d. & n.d. & n.d. & n.d. \\
\hline Usp & n.d. & 0.6 & 17.3 & 37.0 & 40.7 & n.d. & 1.2 & 6.1 & 27.7 & 32.9 \\
\hline Mgt & 8.1 & 41.0 & 70.9 & 5.55 & 49.9 & 62.7 & 96.7 & 90.0 & 56.5 & 56.3 \\
\hline Il & 1.2 & 6.2 & 9.8 & 5.2 & $3.7 *$ & 22.2 & n.d. & n.d. & 8.3 & n.d. \\
\hline $\mathrm{Sp}$ & 54.5 & 29.4 & 2.0 & 2.3 & 5.2 & 9.6 & 1.8 & 2.7 & 10.5 & 10.3 \\
\hline $\mathrm{Hm}$ & n.d. & n.d. & n.d. & n.d. & n.d. & 5.6 & 0.3 & n.d. & n.d. & 0.5 \\
\hline
\end{tabular}

Table 9. Chemical, crystal-chemical and minal compositions of the ilmenites of the basalt-andesibaslt complexes of the Gochas and the Azykh depressions

\begin{tabular}{|c|c|c|c|c|c|}
\hline & \multicolumn{3}{|c|}{ Gochas depression } & \multicolumn{2}{|c|}{ Azykh depression } \\
\hline $\begin{array}{l}\text { № samples } \\
\text { components. }\end{array}$ & 3 & 4 & 5 & 1 & 2 \\
\hline $\mathrm{TiO}_{2}$ & 49.36 & 48.68 & 48.72 & 48.82 & 48.80 \\
\hline $\mathrm{Al}_{2} \mathrm{O}_{3}$ & 1.10 & 0.64 & 0.73 & 0.46 & 0.58 \\
\hline $\mathrm{V}_{2} \mathrm{O}_{3}$ & 0.18 & 0.44 & 0.38 & 0.82 & 0.94 \\
\hline $\mathrm{Cr}_{2} \mathrm{O}_{3}$ & 0.16 & 0.23 & 0.24 & 1.75 & 1.64 \\
\hline $\mathrm{Fe}_{2} \mathrm{O}_{3}$ & 5.28 & 6.51 & 6.53 & 4.05 & 3.74 \\
\hline $\mathrm{FeO}$ & 41.97 & 40.50 & 40.48 & 42.71 & 42.35 \\
\hline $\mathrm{MnO}$ & 1.38 & 2.63 & 2.71 & 0.64 & 0.68 \\
\hline $\mathrm{MgO}$ & 0.52 & 0.33 & 0.43 & 0.43 & 0.66 \\
\hline$\sum$ & 99.95 & 99.96 & 100.22 & 99.68 & 99.43 \\
\hline $\mathrm{t}^{\circ} \mathrm{C}$ & 820 & 920 & 1050 & 870 & 900 \\
\hline $\mathrm{fo}_{2}$ & -12 & -11.3 & -9.2 & -12.5 & -11.8 \\
\hline $\mathrm{Ti}$ & 0.931 & 0.922 & 0.920 & 0.928 & 0.927 \\
\hline $\mathrm{Al}$ & 0.033 & 0.019 & 0.021 & 0.014 & 0.017 \\
\hline $\mathrm{V}$ & 0.003 & 0.009 & 0.008 & 0.017 & 0.019 \\
\hline $\mathrm{Cr}$ & 0.003 & 0.004 & 0.005 & 0.035 & 0.033 \\
\hline $\mathrm{Fe}^{+3}$ & 0.100 & 0.124 & 0.123 & 0.077 & 0.071 \\
\hline $\mathrm{Fe}^{+2}$ & 0.880 & 0.853 & 0.851 & 0.903 & 0.895 \\
\hline $\mathrm{Mn}$ & 0.029 & 0.056 & 0.057 & 0.010 & 0.015 \\
\hline $\mathrm{Mg}$ & 0.019 & 0.012 & 0.016 & 0.016 & 0.024 \\
\hline $\mathrm{MnTiO}_{3}$ & 2.1 & 5.2 & 5.3 & 0.9 & 1.4 \\
\hline $\mathrm{MgTiO}_{3}$ & 1.8 & 1.1 & 1.5 & 1.5 & 2.2 \\
\hline $\mathrm{Fe} \mathrm{TiO}_{3}$ & 82.5 & 79.2 & 76.3 & 84.1 & 82.7 \\
\hline $\mathrm{Fe}_{2} \mathrm{O}_{3}$ & 13.0 & 14.5 & 14.9 & 13.4 & 13.7 \\
\hline
\end{tabular}

Therefore, iron-titanium oxide minerals in the compound of highly titanium differentiates of the Khojavand, Azykh and Gochas depression have in general crystallized in an oxidative environment. Thus, crystallization of moderately titanium magnetite occurred before or at the same time with clinopyroxene.

Unlike the intrusive teshenites, the rocks of trachybasalt-trachydolerite complex crystallized in a comparatively reductive environment. At the same time, impregnations of the main plagioclase underwente crystallization either together or later than titanomagnetite.

The calculated Curie points $(\mathrm{Tc}=230,250)$ and therefore ulvospinel molecules $\left(\mathrm{Usp}_{48-53}\right)$ in the composition of the titanomagnetite indicate that intrusive teshenites crystallized in relatively deeper conditions, because of which the relatively titanium magnetites are less enriched with alumina.

Phenocrysts of moderately titanium magnetites in andesibasalts and andesites developed at deeper intermediate sites.

\section{Conclusions:}

1. Based on calculated and experimental Curie points and physical-chemical parameters ( $\mathrm{t}, \mathrm{fo}_{2}, \mathrm{H}$ и др.), and also the determined variations of compositions, we may state that crystallization of iron-titanium oxide minerals occurred at intermediate sites of various 
depths. At the same time, during the Late Coniacian and Early Santonian periods, in the Gazakh, Agjakand and Agdara depressions, there continued stable depression, which caused poorly differentiated portions of olivine basalt melt to localize at deeper intermediate sites, from which the highly titanous magnetite crystallized. At the boundary of the Upper Santonian-Lower Campanian periods, the activity of magma-providing faults within the Gazakh Depression significantly weakened. Therefore, within this fault, near-the-surface intermediate sites formed, the presence of which may be indicated by spatial division of volcanic centers, composed of rhyodacites, perlite, andesite and dacite.

2. Unlike the Gazakh depression, within the Agjakand and Agdara depressions, the process of compression manifested more intensely, causing thermal impact of hot basalt melt with above embedded arkose containing sandstones. This caused those sandstones to melt and led to generation of pallingenic rhyodacites and dacites.

3. Within the Khojavand and Azykh depressions, in the conditions of depression during the Early Santonian Age, a poorly differentiated trachybasalttrachydolerite complex formed. The differentiates of this

\section{References}

Akhundov, F.A., 2003. Pozdnemelovye vulkanicheskie formacii Malogo Kavkaza. [Late Cretaceous volcanic formations of the Lesser Caucasus].Baku, 232.

Anderson, A.T., 1968. Oxidation of the La Blanche Lake titaniferrous magnetite deposit, Quebec. Journal of Geology, 76, 5, 528-547.

Avdeev, A.G., Ostroumova, A.S., Center, I. Ja., Shafiev, H.I., 1989. Epilejcitovye tefrity verhnemelovogo vulkanicheskogo kompleksa Martuninskogo sinklinorija (Juzhnyj Karabah). [Epileucite tephrites of the Upper Cretaceous volcanic complex of the Martuni Sinclinorium (Southern Caucasus)] Bulletin of the Moscow Society of Naturalists. Geological Department, 64, 3, 86-96. (In Russian)

Buddington, A.F., Lindsley, D.H., 1964. Iron-titanium oxide minerals and synthetic equivalents. Journal of Petrology, 5, 310-357.

Carmichael, I.S.E., 1967. The iron-titanium oxides of salic volcanic rocks and their associated ferromagnesian silicates. Contributions to Mineralogy and Petrology, 14 (1), 36-64.

Creer, K.M. and Ibbetson, J.D., 1970. Electron microprobe analyses and magnetic properties of non-stoichiometric titanomagnetites in basaltic rocks. Geophys. J. R. astr. Soc., 21, 485-511.

Fabries, J., 1979. Spinel-olivine geothermometry in peridotites form ultramafic complexes. Contributions to Mineralogy and Petrology, 69, 329-336.

Genshaft, Ju.S., Celmovich, V.A., Gapeev, A.K., Solodovnikov, G.M. 1999. Znachenie Fe-Ti oksidnyh complex include moderately titanous titanomagnetite. In relatively oxidative conditions during the Late Santonian Age, the differentiates of the tephriteteshenite complex crystallized. In the same place, due to slowing of the crystallization of plagioclase, moderately titanium magnetite in the tephrites was significantly enriched with alumina. In the composition of intrusive teshenites, crystallization was undergone by ulvospinel titanomagnetite.

4. In the Gochas depression, crystallization in the basalts and andesibasalts was first undergone by clinopyroxene. In the compound of rocks of subalkaline series, more titanium magnetite crystallized together with clinopyroxene.

5. During the crystallization of the Late Cretaceous volcanic complexes at intermediate sites of various depths, the most melanocratic components of magmatic melts formed at relatively higher temperatures and restorative conditions $\left(\mathrm{t}=1030-1080^{\circ} \mathrm{C}, \mathrm{fo}_{2}=-9,2-10\right)$. Sedimentary melts, which by composition correspond to andesitess, dacites and rhyolites, were developed in comparatively low thermodynamic conditions $(\mathrm{t}=758-$ $980{ }^{\circ} \mathrm{C}$, fo ${ }_{2}=-5.9-7.2$ ), accompanied by oxidized volatile components.

mineralov v naukah o Zemle. Vestnik OGGGG RAN, 1(7), 3-12. (In Russian)

Genshaft, Ju.S, Celmovich, V.A, Gapeev, A.K. 2003. Kristallizacija vysokotitanistoj ferroshpineli v P-T uslovijah verhnej mantii [Crystallization of highly titanous ferrospinel in P-T conditions of the upper mantle]. Fizika Zemli, 3, 71-75. (In Russian)

Geologija Azerbajdzhana. 2005. Magmatizm. V. III.

Karpova, O.V., 1974. Titanomagnetitovye rudy Juzhnogo Urala [Titanomagnetite ores of the Southern Ural]. Moskva: Nauka, 152 c. (In Russian)

Karjakin, Ju.V., 1989. Geodinamika formirovanija vulkanicheskih kompleksov Malogo Kavkaza [Geodynamics of the development of the volcanic complexes of the Lesser Caucasus] Moskva: Nauka, 150. (In Russian)

Kawai, N., 1956. Exsolution of titanomagnetites and its effect on rock-magnetism. Proceedings of the Jap.Acad., 32, 464-468.

Lepage, L.D., 2003. ILMAT: an excel worksheet for ilmenite-magnetite geothermometry and geobarometry. Comput. Geosci. 29 (5), 673-678.

Lindsley, D. H. \& Spencer, K.J., 1982. Fe-Ti oxide geothermometry: Reducing analyses of coexisting Timagnetite (Mt) and ilmenite (Ilm) abstract AGU 1982 Spring Meeting Eos Transactions. American Geophysical Union, 63, 18, 471.

Osborn, E.F., 1983. On the significance of the spinel phase in subalkaline volcanic magmas. Memoir of the geological society of China. 5, 1-12. 
Ostroumova, A.S., Center, I. Ja., Avdeev, A.T., Bugrova, J.M. 1991. Pozdnemelovye vulkanicheskie serii Gochasskogo progiba (Malyj Kavkaz) [Late Creataceous volcanic series of the Gochas depression (Lesser Caucasus)]. Sovetskaja geologija, 7, 65-72. (In Russian)

Sack, R.O., Carmicahael, I.S., Rivers, S.E., Ghiorso, M.S., 1980. Ferric-ferous equilibria in natural silicate liquids at 1 bar. Contributions to Mineralogy and Petrology, 75, 369-376.

Stormer, J.C., 1983. The effects of recalculation on estimates of temperature and oxygen fugacity from analyses of multicomponent iron-titanium oxides. American Mineralogist, 68, 5-6, 586-594.

Mamedov, M.N., 1999. Petrologija i geohimija pozdnemelovyh i jeocenovyh magmaticheskih for-macij Malogo Kavkaza i Talysha [Petrology and geochemistry of the Late Cretaceous and Eocene magnetic formations of the Lesser Caucasus and Talysh]. Baku: Nafta-press, 400. (In Russian)

Mamedov, M.N., Babaeva, G.D., Sadygov, N.M., 2012. Klinopirokseny trahibazalt-trahidoleritovogo i tefritteshenitovogo kompleksov Hodzhavendskogo progiba. [Clinopyroxene trachybasalt-trachydolerite and tephriteteshenite complexes of the Khojavand depression] Otechestvennaja geologija, 6, 48-55. (In Russian)

Mamedov, M.N., Babaeva, G.D., Panahi, K.A., Sadygov, N.M., Kerimov, V.M., 2013. Indikatornye znachenija zhelezo-titan oksidnyh mineralov v processe kristallizacii porod trahibazalt-trahidoleritovogo i tefrit-teshenitovogo kompleksov Hodzhavendskogo progiba Malogo Kavkaza. [Indicative values of the iron titanium oxide minerals in the process of crystallization of rocks of trachybasalt-trachydolerite and tephrite-teshenite complexes of the Khojavand depression of the Lesser Caucasus] News of Baku University, series of natural sciences, 3, 76-85.
Mamedov, M.N., Panahi, K.A., Babaeva, G.D., Sadygov, N.M., 2015. Petrogeneticheskie znachenija shpinelidov v processe kristallizacii porod trahibazalt-trahidoleritovogo i tefrit-teshenitovogo kompleksov Hodzhavendskogo progiba Malogo Kavkaza. [Petrogenetic values of spinelides in the processes of crystallization of rocks of trachybasalt-trachydolerite and tephrite-teshenite complexes of the Khojavand depression of the Lesser Caucasus] News of Baku University, series of natural sciences, 3, 82-96. (In Russian)

Nagata, T., 1965. Magnetizm gornyh porod [Magnetism of rocks]. Moscow: Mir, 347. (In Russian)

Pecherskij, D.M. 1975. Magnetizm i uslovija obrazovanija izverzhennyh gornyh porod. [Magnetism and node formations of eruptive rocks] Moscow: Nauka, 1975, 288. (In Russian)

Pecherskij, D.M. 1985. Petromagnetizm i paleomagnetizm. [Petromagnetis and paeomagnetism] Moscow: Nauka, 128. (In Russian)

Shikhalibejli, H. Sh., 1994. Geologija i poleznye iskopaemye Nagornogo Karabaha Azerbajdzhana [Geology and fossils of the Nagorno-Karabakh of Azerbaijan]. Baku: Elm, 281. (In Russian)

Zubov, A.G., Bazanova, L.I., Ananev, V.V., Ovsjannikov, A.A., Zhidkov, G.V., 2015. O primenenii titanomagnetitovogo metoda dlja opredelenija glubiny magmaticheskogo ochaga na primere nekotoryh kamchatskih vulkanov [On use of titanomagnetite method of determining depth of magmetite sites on the example of some Kamchatka volcanoes]. Material of annual conference Volcanism and Processes Related To It, dedicated to Day of Volcanologist, 138-147. (In Russian) 